Nature Reviews Immunology | AOP, published online 21 January 2008; doi:10.1038/nri2255

INNATE IMMUNITY

\section{TAMing inflammation}

Toll-like receptors (TLRs) are crucial for detecting infection and activating the innate and adaptive immune systems. However, sustained TLR stimulation can result in chronic inflammation and is associated with the development of certain autoimmune diseases. Now, Lemke and colleagues have identified a new signalling feedback loop through the TAM (TYRO3, AXL and MER) receptor family, which broadly inhibits both TLR signalling pathways and TLR-induced cytokine signalling pathways. They suggest that this could provide a mechanism through which the immune system self-regulates.

The TAM receptor family comprises three receptor tyrosine kinases - TYRO3, AXL and MER - that can be activated by binding to two closely related ligands, growth-arrest-specific 6 (GAS6) and protein $\mathrm{S}$. The authors showed that stimulation of dendritic cells (DCs) from mice that were deficient in all three TAM receptors with various TLR ligands resulted in the production of elevated levels of proinflammatory cytokines compared with wild-type DCs. Similarly, preincubation of wild-type DCs with either GAS6 or protein S inhibited TLR-induced cytokine production.

The authors next showed that the activation of TAM receptors blocked several TLR-induced signalling events, including nuclear factor- $\kappa \mathrm{B}$ $(\mathrm{NF}-\kappa \mathrm{B})$ activation. Furthermore, suppressor of cytokine signalling 1

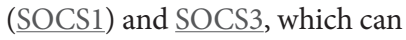
inhibit TLR signalling as well as cytokine signalling, were both induced in response to TAM-receptor stimulation in a signal transducer and activator of transcription 1 (STAT1)-dependent manner, and STAT1 activation was necessary for the inhibition of TLR-induced cytokine production.

STAT1 is commonly associated with signalling downstream of the type I interferon receptor (IFNAR). Further analysis showed that, following TAM-receptor activation, DCs deficient in IFNAR failed to activate STAT1, did not upregulate SOCS protein expression and produced normal levels of cytokines in response to TLR signalling. In addition, they found that signalling through IFNAR upregulated AXL expression and that it associated with the R1 chain of IFNAR. This observation suggests that the TAM receptors can hijack IFNAR-induced STAT1 activation to suppress TLR-induced cytokine production through SOCS proteins.

These findings reveal a new selfregulating cycle in the innate immune system, whereby TLR-induced

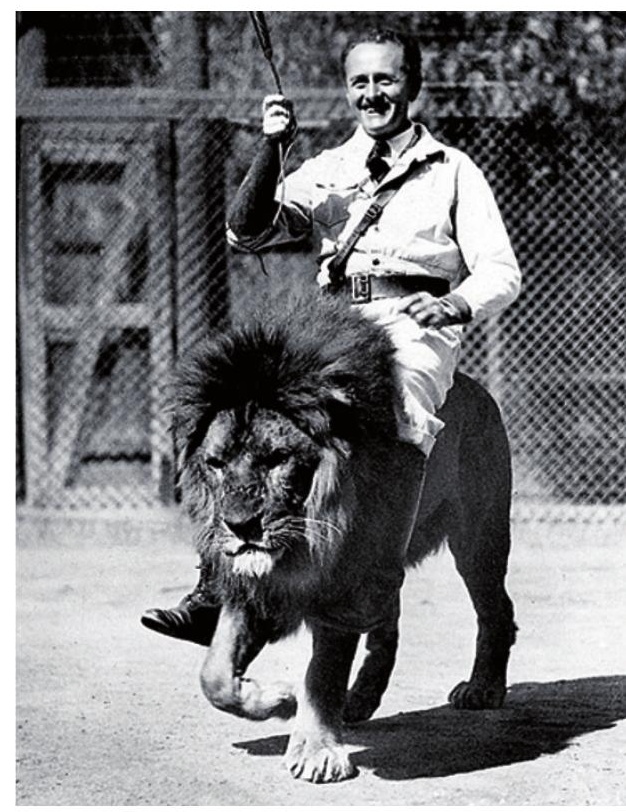

activation of the IFNAR-STAT1 pathway is used to induce the expression of the TAM receptors, which then co-opt the same pathway to initiate an intrinsic negative feedback loop that inhibits both TLR- and cytokine-driven responses. These findings have important implications for the resolution of inflammation, and inhibitors of the TAM receptor signalling pathway could provide attractive candidates for improved vaccine adjuvants.

Jennifer Middleton Intern, Nature Publishing Group

ORIGINAL RESEARCH PAPER Rothlin, C. V. et al TAM receptors are pleiotropic inhibitors of the innate immune response. Nature $\mathbf{1 3 1}$, 1124-1136 (2007) 